\title{
COINCIDENCES AND FIXED POINTS IN LOCALLY G-CONVEX SPACES
}

\author{
P.J. WATSON
}

\begin{abstract}
A new coincidence point theorem is proved for a pair of multivalued mappings operating between $G$-convex spaces. From this theorem, a generalisation of the classical Fan-Glicksberg fixed point theorem is established.
\end{abstract}

\section{INTRODUCTION}

In recent years many researchers have been interested in various notions of convexity on topological spaces which do not rely on a linear structure of the underlying space. The first work in this direction may be Aronszajn and Panitchpakdi [1] where the authors introduced a convexity structure on metric spaces; hyperconvex metric spaces. Subsequently this property has been found to be important in the study of nonexpansive mappings, see $[6,14,15]$.

Some time later Horvath $[9,10,11]$ defined a convexity structure in topological spaces and proved several important results in the theory of nonlinear analysis. The structure determining convexity in this space is a multivalued monotone operator mapping the finite subsets to contractible subsets of the topological space. Note that a contractible set in a topological space is one in which the identity map, restricted to the set in question, is homotopic to a constant map. This structure replaces the convex hull in vector spaces. Such a space has since been called an $H$-convex space (or simply $H$ space) by Bardaro and Ceppitelli [2] where amongst other results, a KKM type theorem is established.

The so-called $G$-convex spaces were introduced in [12] to allow for a convexity structure that need not have contractible values. These spaces generalise the notion of $H$-convexity (see Definition 1 below) as well as hyperconvexity. We refer to $[18,6]$ for further discussion on the relations between these concepts of convexity.

This study examines the existence of coincidence points for multivalued operators acting between different $G$-convex spaces. The first result, Lemma 1, is a fixed point result for the composition of a single valued continuous function and a multivalued operator with $G$-convex values. A selection theorem proved in [16], Theorem 2.1 below, is fundamental

Received 17th September, 1998

Copyright Clearance Centre, Inc. Serial-fee code: 0004-9729/99 \$A2.00+0.00. 
in the proof, and its use replaces linear approximation arguments used when the ambient space is linear (see for example [8, Lemma 2]). From this, a coincidence point theorem is proved and then a fixed point theorem which generalises the classical Fan-Glicksberg fixed point theorem. This study concludes with a fixed point theorem in which the compactness condition on the space is relaxed.

It should be noted that Yuan [18] has generalised the Fan-Glicksberg fixed point theorem for multifunctions with acyclic values, and in Ding and Tarafdar [4], a coincidence point theorem has been proved (in $H$-spaces) for a pair of multifunctions, one of which has acyclic values. The emphasis of this work is to study multifunctions with $G$ convex values instead of acyclic values. Therefore the results established here are proved by different means and they do not compare with the results in $[18,4]$.

\section{2. $G$-CONVEX Spaces}

First we elucidate the notations and definitions used in this paper. Let $X$ be a set. $2^{X}$ denotes the family of all nonempty subsets of the space $X$ and $\mathcal{F}(X)$ denotes the family of nonempty finite subsets of $X . \Delta_{n}$ is the standard $n$-dimensional simplex with verticies $e_{0}, \ldots, e_{n}$ where $e_{0}=0$ and $e_{i}$, for $i=1, \ldots, n$, is the $i$-th unit vector in $\mathbb{R}^{\mathrm{n}}$; that is, $\Delta_{n}=\operatorname{co}\left\{e_{0}, \ldots, e_{n}\right\}$. If $a_{0}, \ldots, a_{n}$ are points in some vector space $X$, then $a_{0} \ldots a_{n}$ will denote the simplex with verticies $a_{0}, \ldots, a_{n}$. Let $X$ and $Y$ be topological spaces. A multifunction $T: X \rightarrow 2^{Y}$ is said to be upper semicontinuous if $T^{-1}(C)=\{x \in X: T(x) \cap C \neq \emptyset\}$ is closed in $X$ whenever $C$ is closed in $Y$.

The following definition originally appeared in [12].

DEFINITION 1. A generalised convex, or $G$-convex space $(X, D ; \Gamma)$ is a topological space $X$, a nonempty subset $D$ of $X$ and a function $\Gamma: \mathcal{F}(X) \rightarrow 2^{X}$ with the following properties:

1. for any $A, B \in \mathcal{F}(X)$ with $A \subset B$, we have $\Gamma(A) \subset \Gamma(B)$;

2. for any $A \in \mathcal{F}(X)$ with elements $a_{0}, \ldots, a_{n}$ there exists a continuous function $\psi: \Delta_{n} \rightarrow \Gamma(A)$ such that for each $0 \leqslant i_{0}<\cdots<i_{k} \leqslant n$ it follows that

$$
\psi\left(\operatorname{co}\left\{e_{i_{0}}, \cdots, e_{i_{k}}\right\}\right) \subset \Gamma\left(\left\{a_{i_{0}}, \cdots, a_{i_{k}}\right\}\right) .
$$

$(X, \Gamma)$ is an $H$-space when $D=X$, condition 1 is satisfied and the operator $\Gamma$ has contractible values. It has been shown in $[10$, Theorem 1] that such an operator satisfies condition 2.

A subset $K$ of a $G$-convex space $(X, D ; \Gamma)$ is said to be $G$-convex if, for any $A \in$ $\mathcal{F}(K \cap D), \Gamma(A) \subset K$. Note that the intersection of $G$-convex sets remains $G$-convex. The $G$-convex hull of a subset $Y$ of a $G$-convex space, denoted $G$-co $(Y)$, is defined to be the intersection of all $G$-convex sets containing the set $Y$. So the $G$-convex hull of $Y$ is the smallest $G$-convex set containing $Y$, which is evidently $G$-convex. Further properties 
of $G$-convex spaces and sets can be found in [12] and [16]. In this study, the set $D$ in the definition of $G$-convex will be all of $X$ and $(X, X ; \Gamma)$ will be denoted $(X ; \Gamma)$.

The following definition relates the $G$-convex sets with the topology of $X$, and it generalises the concept of a locally convex topological vector space.

DEFINITION 2. A G-convex space $(X ; \Gamma)$ is said to be a locally $G$-convex uniform space if $X$ is a uniform space with uniformity $\mathcal{U}$ having base $\beta$ of open symmetric entourages such that each $W \in \beta$ satisfies the property that

$$
W(x)=\{y \in X:(x, y) \in W\}
$$

is G-convex.

An arbitrary entourage satisfying this property will be said to be $G$-convex.

An alternative definition of local $G$-convexity is to assume that for any $W \in \beta$, $W(K)=\{x \in X:(y, x) \in W$ for some $y \in K\}$ is $G$-convex whenever $K$ is $G$-convex. A locally $G$-convex space satisfying this property has fewer $G$-convex sets than one satisfying Definition 2. This follows as Definition 2 implies each singleton is $G$-convex (simply note $\{x\}=\bigcap_{V \in \beta} V(x)$ and the intersection of $G$-convex sets is $G$-convex), whereas the second notion does not necessarily imply this. Although the alternative definition gives rise to a more general space, it may be the case that there are fewer multifunctions with $G$-convex values (for example, single valued functions may not have $G$-convex values). Thus we restrict our analysis to locally $G$-convex spaces as in Definition 2 . Note that both concepts coincide if the $G$-convex space $(X ; \Gamma)$ is such that $\Gamma(x)=\{x\}$ for all $x \in X$.

It is well know that in uniform spaces, the closure of a set $K \subset X$ is given by

$$
\bar{K}=\bigcap\{V(K): V \in \beta\}
$$

where $\beta$ is any base for $\mathcal{U}$. It follows that in locally $G$-convex uniform spaces, the closure of a $G$-convex set, being the intersection of $G$-convex sets, is $G$-convex.

The following selection theorem is a weaker formulation of [16, Theorem 2.4], though sufficient for our purpose.

TheOREM 2.1. Let $X$ be a compact topological space and $(Y ; \Gamma)$ a $G$-convex space. Suppose $T: X \rightarrow 2^{Y}$ satisfies

1. $T(x)$ is $G$-convex for all $x \in X$;

2. for each $x \in X$ there exists $y \in Y$ such that $x \in \operatorname{int}\left(T^{-1}(y)\right)$.

Then there exists $A \in \mathcal{F}(Y)$ and continuous functions $g: \Delta_{n} \rightarrow Y$ and $\phi: X \rightarrow \Delta_{n}$, where $n+1=|A|$, such that the composition $f=g \circ \phi$ is a continuous selection of $T$; that is, $f(x) \in T(x)$ for all $x \in X$.

\section{A Coincidence Theorem}

The first result is the G-convex version of [8, Lemma 2] and is similar to the fixed point theorems of Eilenberg and Montgomery [5], Gòrniewicz [7] and Shioji [13], although 
the setting is a locally $G$-convex space and the multifunction has $G$-convex values rather than contractible or acyclic values.

Lemma 1. Let $(X ; \Gamma)$ be a compact locally $G$-convex uniform space. Suppose $p: X \rightarrow \Delta_{n}$ is continuous and $q: \Delta_{n} \rightarrow 2^{X}$ is upper semicontinuous with compact $G$-convex values. Then $p \circ q: \Delta_{n} \rightarrow 2^{\Delta_{n}}$ has a fixed point.

Proof: For $k=1,2, \ldots$, denote by $S^{k}$ the $k$-th barycentric subdivision of the simplex $\Delta_{n}$. For each $k$ define a multivalued mapping $T_{k}: \Delta_{n} \rightarrow 2^{X}$ by

$$
T_{k}(v)=G \text {-со }\left\{\bigcup_{i=0}^{r} q\left(a_{k}^{i}\right)\right\}
$$

where $a_{k}^{i}$, for $i=0, \ldots, r, 0 \leqslant r \leqslant n$, are the verticies of the simplex in $S^{k}$ of least dimension containing the point $v$. The values of $T_{k}$ are clearly $G$-convex.

We prove condition 2 of Theorem 2.1 is satisified for $T_{k}$. So we show each $v \in \Delta_{n}$ belongs to the interior of $T_{k}^{-1}(y)$ for some $y \in X$. To this end, let $v \in \Delta_{n}$ be arbitrary. For $a_{k}^{0} \ldots a_{k}^{r}$ the simplex in $S^{k}$ of least dimension containing $v$, choose $\varepsilon>0$ such that $\varepsilon<\operatorname{dist}\left(v, \Lambda_{k}\right)$ for all simplexes $\Lambda_{k} \in S^{k}$ with $v \notin \Lambda_{k}$. We claim the open ball $B_{\varepsilon}(v)$ in $\Delta_{n}$ is a subset of

$$
\Phi=\bigcup\left\{\Lambda_{k}^{n} \in S^{k}: a_{k}^{0} \ldots a_{k}^{r} \text { is a face of } \Lambda_{k}^{n} \text { and } \operatorname{dim} \Lambda_{k}^{n}=n\right\} .
$$

To see this, suppose $z$ is not an element of $\Phi$. Then $z \in \Delta_{n} \backslash \Lambda_{k}^{n}$ for all $n$-dimensional $\Lambda_{k}^{n} \in S^{k}$ having $a_{k}^{0} \ldots a_{k}^{r}$ as a face. Hence $z$ belongs to an $n$-dimensional simplex $\widehat{\Lambda}_{k}^{n}$ and $a_{k}^{0} \ldots a_{k}^{r}$ is not a face of $\widehat{\Lambda}_{k}^{n}$. Either $a_{k}^{0} \ldots a_{k}^{r} \cap \widehat{\Lambda}_{k}^{n}=\emptyset$ or not. In the first case it immediately follows that $v \notin \widehat{\Lambda}_{k}^{n}$ so $z \notin B_{\varepsilon}(v)$ from the definition of $\varepsilon$. If $a_{k}^{0} \ldots a_{k}^{r} \cap \widehat{\Lambda}_{k}^{n} \neq \emptyset$ then the intersection is a face common to both. As $a_{k}^{0} \ldots a_{k}^{r}$ is not a face of $\widehat{\Lambda}_{k}^{n}$, the intersection must be a simplex of dimension strictly less than $r$. As $r$ is the smallest integer such that $v \in a_{k}^{0} \ldots a_{k}^{r}$ then $v \notin \widehat{\Lambda}_{k}^{n}$ so again $z \notin B_{\varepsilon}(v)$.

Thus the inclusion $B_{\varepsilon}(v) \subset \Phi$ has been established. This implies that for each $w \in B_{\varepsilon}(v), T_{k}(v) \subset T_{k}(w)$ by the definition of $T_{k}$ and $\Phi$. By choosing $y \in T_{k}(w)$, it follows that $B_{\varepsilon}(v) \subset T_{k}^{-1}(y)$ and condition 2 of Theorem 2.1 is satisfied.

By Theorem 2.1 there exists a continuous selection $f_{k}$ of $T_{k}$. The composition $p \circ f_{k}$ : $\Delta_{n} \rightarrow \Delta_{n}$ is continuous and so by Brouwer's fixed point theorem, there exists $v_{k} \in \Delta_{n}$ such that $v_{k}=p\left(f_{k}\left(v_{k}\right)\right)$. Let $x_{k}=f_{k}\left(v_{k}\right)$. As $X$ is compact we may assume the net $x_{k}$ converges to $x_{0} \in X$. As $p$ is continuous, $v_{k}=p\left(x_{k}\right) \rightarrow p\left(x_{0}\right)=v_{0}$. We claim $x_{0} \in q\left(v_{0}\right)$ so that $v_{0}$ is a fixed point of the multivalued composition $p \circ q$.

As $q\left(v_{0}\right)$ is closed it is enough to show $x_{0} \in V\left(q\left(v_{0}\right)\right)$ for any $V$ in any base for the uniformity $\mathcal{U}$. So let $V$ be a fixed element of some base for the uniformity. As all the closed symmetric entourages form a base for $\mathcal{U}$, there exists a closed symmetric entourage $W \subset V$. Similarly as all the open symmetric $G$-convex entourages form a base for the 
uniformity, there exists an open symmetric $G$-convex $W_{1} \subset W$. Therefore $W_{1}\left(q\left(v_{0}\right)\right)$ is an open $G$-convex neighbourhood of $q\left(v_{0}\right)$. By upper semicontinuity of $q$, there exists a neighbourhood $N\left(v_{0}\right)$ such that $q(v) \subset W_{1}\left(q\left(v_{0}\right)\right)$ for all $v \in N\left(v_{0}\right)$.

For each barycentric subdivision $S^{k}$ of $\Delta_{n}$ there exists an $n$-simplex $a_{k}^{0} \ldots a_{k}^{n}$ containing the point $v_{k}$ and moreover $a_{k}^{i} \rightarrow v_{0}$ for each $i=0,1, \ldots, n$ as $k \rightarrow \infty$. For $k$ sufficiently large, $a_{k}^{i} \in N\left(v_{0}\right)$ for each $i=0,1, \ldots, n$ and

$$
x_{k}=f_{k}\left(v_{k}\right) \in G \text {-co }\left\{\bigcup_{i=0}^{n} q\left(a_{k}^{i}\right)\right\} .
$$

As $W_{1}\left(q\left(v_{0}\right)\right)$ is $G$-convex and $a_{k}^{i} \in N\left(v_{0}\right)$ it follows that

$$
x_{k} \in G \text {-co }\left\{\bigcup_{i=0}^{n} q\left(a_{k}^{i}\right)\right\} \subset W_{1}\left(q\left(v_{0}\right)\right) \subset W\left(q\left(v_{0}\right)\right) .
$$

This implies $x_{0} \in W\left(q\left(v_{0}\right)\right) \subset V\left(q\left(v_{0}\right)\right)$ as $W$ is closed and $q\left(v_{0}\right)$ is compact. As $V$ is arbitrary, $x_{0} \in q\left(v_{0}\right)$.

Using this, the following coincidence point theorem is established.

THEOREM 3.1. Let $(X ; \Gamma)$ be a compact locally $G$-convex space and $(Y ; \Sigma)$ an arbitrary $G$-convex space. Suppose $F: X \rightarrow 2^{Y}$ is such that

1. $F(x)$ is $G$-convex for all $x \in X$;

2. $F^{-1}(y)$ contains an open set $O_{y}$ (which may be empty for some $y$ );

3. $\bigcup_{y \in Y} O_{y}=X$.

Then for each upper semicontinuous $g: Y \rightarrow 2^{X}$ with compact $G$-convex values there exists a coincidence point; that is, a point $x_{0} \in X$ such that

$$
F\left(x_{0}\right) \cap g^{-1}\left(x_{0}\right) \neq \emptyset \text {. }
$$

Proof: By Theorem 2.1 there exists $n \in \mathbb{N}$ and continuous maps $h: \Delta_{n} \rightarrow Y$ and $\phi: X \rightarrow \Delta_{n}$ such that $f=h \circ \phi$ is a continuous selection of $F$. The composition $g \circ h$ : $\Delta_{n} \rightarrow 2^{X}$ is upper semicontiuous with compact $G$-convex values. From Lemma 1 there exists $v_{0} \in \Delta_{n}$ with $v_{0} \in \phi\left(g\left(h\left(v_{0}\right)\right)\right)$. Letting $y_{0}=h\left(v_{0}\right)$, we have $y_{0} \in h\left(\phi\left(g\left(y_{0}\right)\right)\right)$; that is, $y_{0}=h(\phi(z))=f(z)$ for some $z \in g\left(y_{0}\right)$. Hence $y_{0} \in F(z) \cap g^{-1}(z)$ as required.

\section{FIXed PoInts}

As an application of Theorem 3.1, the Fan-Glicksberg fixed point theorem is generalised to locally $G$-convex spaces as follows.

THEOREM 4.1. Let $(X ; \Gamma)$ be a compact locally $G$-convex uniform space. Then any upper semicontinuous $g: X \rightarrow 2^{X}$ with closed $G$-convex values has a fixed point. 
Proof: For $W \in \beta$ arbitrary, so $W$ is an open symmetric $G$-convex entourage, define a multifunction $F_{W}: X \rightarrow 2^{X}$ by $F_{W}(x)=W(x)$. It is clear that $F_{W}$ has $G$ convex values. Also $F_{W}^{-1}(y)=W^{-1}(y)=W(y)$ as $W$ is symmetric. By Theorem 3.1 there exists $x_{W} \in X$ such that $g\left(x_{W}\right) \cap F_{W}^{-1}\left(x_{W}\right) \neq \emptyset$. Let $z_{W}$ be an element of this intersection. Thus $x_{W} \in F_{W}\left(z_{W}\right) \subset F_{W}\left(g\left(x_{W}\right)\right)=W\left(g\left(x_{W}\right)\right)$.

For each $W \in \beta$, let $H_{W}=\{x \in X: x \in \bar{W}(g(x))\}$ which is nonempty by the above arguments. Moreover $H_{W}$ is closed. Indeed, let $\left\{x_{\delta}\right\}$ be a net in $H_{W}$ converging to $x_{0}$. Then there exists a net $\left\{u_{\delta}\right\}$ such that $x_{\delta} \in \bar{W}\left(u_{\delta}\right)$ and $u_{\delta} \in g\left(x_{\delta}\right)$. As $X$ is compact, without loss of generality we may assume $u_{\delta} \rightarrow u_{0} \in X$. As $g$ is upper semicontinuous, it has a closed graph so $u_{0} \in g\left(x_{0}\right)$. Also $\left(x_{\delta}, u_{\delta}\right) \in \bar{W}$ so $\left(x_{0}, u_{0}\right) \in \bar{W}$, that is, $x_{0} \in \bar{W}\left(u_{0}\right) \subset \bar{W}\left(g\left(x_{0}\right)\right)$ and $H_{W}$ is closed.

As any finite intersection of elements in $\beta$ is again an element of $\beta$, the compactness of $X$ implies $\bigcap\left\{H_{W}: W \in \beta\right\} \neq \emptyset$. For $x_{0}$ a member of this intersection, $x_{0} \in \bar{W}\left(g\left(x_{0}\right)\right)$ for all $W \in \beta$. We claim $x_{0}$ is a fixed point of $g$. As in the proof of Lemma 1 , it is enough to show $x_{0} \in V\left(g\left(x_{0}\right)\right)$ for any $V$ in an arbitrary basis for the uniformity $\mathcal{U}$. So let $V$ be arbitrary but fixed. We may choose a closed symmetric entourage $W_{1}$ and a $W_{2} \in \beta$ such that $W_{2} \subset W_{1} \subset V$. Then $x_{0} \in \overline{W_{2}}\left(g\left(x_{0}\right)\right) \subset W_{1}\left(g\left(x_{0}\right)\right) \subset V\left(g\left(x_{0}\right)\right)$, which completes the proof.

This result extends [17, Theorem 2.1] to $G$-convex spaces as well as considering upper semicontinuous rather that continuous multifunctions.

When the domain $X$ is not compact, under stronger conditions for the mapping $g: X \rightarrow 2^{X}$ we have:

ThEOREM 4.2. Let $(X ; \Gamma)$ be a locally $G$-convex space, $D \subset X$ closed and $G$ convex, and $g: D \rightarrow 2^{D}$ upper semicontinuous with compact $G$-convex values. If for some $e \in D$ the following implication holds:

$$
(V=G \text {-co } g(V) \text { or } V \subset g(V) \cup\{e\}) \Rightarrow V \text { is relatively compact }
$$

for any subset $V$ of $D$,

then $g$ has a fixed point.

Proof: In the proof we employ some ideas from the paper of Danes [3]. Define a net $\left\{y_{n}\right\}$ as follows: $y_{0}=e$ and $y_{n+1} \in g\left(y_{n}\right)$. Let $Y=\left\{y_{n}: n \geqslant 0\right\}$. Then $Y \subset g(Y) \cup\{e\}$ so by assumption, $Y$ is relatively compact. The set $Z$ of limit points of $Y$ is therefore nonempty and moreover $Z \subset g(Z)$. Indeed, for arbitrary $z_{0} \in Z$, there exists a subnet $y_{n_{i}} \rightarrow z_{0}, y_{n_{i}} \in Y$. By construction of the net $Y$, we have $\left(y_{n_{i}}, y_{n_{i}-1}\right) \in \operatorname{Graph}\left(\left.g\right|_{\bar{Y}}\right)$ which is compact by the compactness of $\bar{Y}$ and upper semicontinuity of $g$. Therefore $\left(y_{n_{i}}, y_{n_{i}-1}\right) \rightarrow\left(z_{0}, z_{1}\right)$ for some $z_{1} \in Z$. This means $z_{0} \in g\left(z_{1}\right)$ and so $Z \subset g(Z)$.

Let $\Omega$ be the family of all subsets $K \subset D$ such that $Z \subset K$ and $G$-cog$g(K) \subset K$. Then $\Omega \neq \emptyset$ as $D \in \Omega$. Let $V=\bigcap\{K: K \in \Omega\}$, which is nonempty as $Z \subset V$. 
Also $G$-cog$g(V) \subset G$-co $g(K) \subset K$ for all $K \in \Omega$. Therefore $G$-co $g(V) \subset V$ and since $G$-co $g(V) \in \Omega$ is clear, then $V \subset G$-co $g(V)$. Thus $V=G$-co $g(V)$ so by assumption, $V$ is relatively compact. Applying now Theorem 4.1 with $X=\bar{V}$, we conclude the mapping $g$ has a fixed point.

\section{REFERENCES}

[1] N. Aronszajn and P. Panitchpakdi, 'Extensions of uniformly continuous transformations and hyperconvex metric spaces', Pacific J. Math. 6 (1956), 405-439.

[2] C. Bardaro and R. Ceppitelli, 'Some further generalisations of the KnasterKuratowski-Mazurkiewicz theorem and minimax inequalities', J. Math. Anal. Appl. 132 (1998), 484-490.

[3] J. Daneš, 'Some fixed point theorems', Comment. Math. Univ. Carolin. 9 (1968), 223-235.

[4] X.P. Ding and E. Tarafdar, 'Some coincidence theorems and applications', Bull. Austral. Math. Soc. 50 (1994), 73-80.

[5] S. Eilenberg and D. Montgomery, 'Fixed point theorems for multivalued transformations', Amer. J. Math. 68 (1946), 214-222.

[6] K. Goebel and W. A. Kirk, Fixed point theory in metric spaces (Cambridge University Press, Cambridge, 1990).

[7] L. Gònniewicz, 'A Lefschetz-type fixed point theorem', Fund. Math. 88 (1975), 103-115.

[8] C.W. Ha, 'On a minimax inequality of Ky Fan', Proc. Amer. Math. Soc. 99 (1987), 680-682.

[9] C. Horvath, 'Some results on multivalued mappings and inequalities with a generalised convexity structure', in Nonlinear and convex analysis, (B.L. Lin and S. Simons, Editors) (Marcel Dekker, New York, 1987), pp. 96-106.

[10] C. Horvath, 'Contractibility and generalised convexity', J. Math. Anal. Appl. 156 (1991), 341-357.

[11] C. Horvath, 'Extension and selection theorems in topological spaces with a generalised convexity structure', Ann. Fac. Sci. Toulouse Math. 2 (1993), 253-269.

[12] S. Park and H. Kim, 'Admissable classes of multifunctions on generalized convex spaces', Proc. Coll. Nat. Sci. SNU 18 (1993), 1-21.

[13] N. Shioji, 'A further generalisation of the Knaster-Kutatowski-Mazurkiewicz theorem', Proc. Amer. Math. Soc. 111 (1991), 187-195.

[14] R.C. Sine, 'Hyperconvexity and approximate fixed points', Nonlinear Anal. 13 (1989), 863-869.

[15] P.M. Soardi, 'Existence of fixed points of nonexpansive mappings in certain Banach lattices', Proc. Amer. Math. Soc. 73 (1979), 25-29.

[16] K-K. Tan and X-L. Zhang, 'Fixed point theorems in $G$-convex spaces and applications', in The Proceedings of the First International Conference on Nonlinear Functional Analysis and Applications, Kyungnam University, Masan, Korea 1, 1996, pp. 1-19.

[17] E.U. Tarafdar, 'Fixed point theorems in locally $H$-convex uniform spaces', Nonlinear Anal. 29 (1997), 971-978.

[18] G. X-Z. Yuan, 'Fixed Points of upper semicontinuous mappings in locally $G$-convex uniform spaces', Bull. Austral. Math. Soc. 58 (1998), 469-478. 
Department of Mathematics The University of Queensland Queensland 4072

Australia

e-mail: pjw@maths.uq.edu.au 